\title{
Experimental demonstration and numerical study of plasmon-soliton waves
}

\author{
Gilles Renversez $^{1}$, Mahmoud M. R. Elsawy ${ }^{1}$, Mathieu Chauvet $^{2}$, Tintu Kuriakose ${ }^{2}$, Tomaz Halenkovic ${ }^{3}$, \\ Virginie Nazabal ${ }^{4}$, Petr P. Nĕmec ${ }^{3}$ \\ 1. Aix-Marseille Univ, CNRS, Centrale Marseille, Institut Fresnel, Marseille, 13013, France \\ 2. FEMTO-ST Institute, CNRS, Université de Bourgogne Franche-Comté, 25030 Besançon, France \\ 3. Department of Graphic Arts and Photophysics, Faculty of chemical Technology, University of Pardubice, 53210 Pardubice, Czech Republic \\ 4. Institut des sciences chimiques de Rennes, CNRS, Equipe Verres et Céramiques, Université de Rennes 1, 35042 Rennes, France
}

Merging the fields of plasmonics and nonlinear optics authorizes a variety of fascinating and original physical phenomena. In this work, we specifically study the combination of the strong light confinement ability of surface plasmon polaritons (SPP) with the beam self-trapping effect in a nonlinear optical Kerr medium. Although this idea of plasmon-soliton has been the subject of numerous theoretical papers since the eighties [1-4], up to now, no experimental evidence had been revealed yet. In the present study, a proper structure (Fig. 1a) has been designed and fabricated allowing the first experimental demonstration of these hybrid nonlinear waves merging spatial solitons and SPP. To be able to trigger the nonlinearity at moderate light power and simultaneously to allow propagation over several millimeters, a multilayered dielectric-metal structure was first designed by modeling [5]. It consists of a five-layer planar geometry (Fig. 1a) made from a silicon wafer with a thick silica layer covered by a chalcogenide layer (green) that is covered with a $10 \mathrm{~nm}$ silica layer followed by a gold film (yellow). Performed numerical simulations show that the main nonlinear TM modes of the designed planar waveguide exhibits a strongly enhanced Kerr self-focusing thanks to the plasmonic effect compared to the main TE nonlinear mode. The experimental analysis consists in injecting a typical $4 \times 30 \mu \mathrm{m}^{2}$ (FWHM) elliptical beam at $1.55 \mu \mathrm{m}$ from a femtosecond laser into the $5 \mathrm{~mm}$ long structure. The output beam distribution evolution is then monitored versus input light intensity. Fig. 1 shows the large trapping enhancement observed for a TM mode in presence of the plasmonic structure (Fig. 1d,e) compared to configurations (Fig. 1b, c and Fig. 1f, g) where the plasmonic effect is absent. For an input intensity of $1.17 \mathrm{GW} / \mathrm{cm}^{2}$ the beam is self-confined to a $12 \mu \mathrm{m}$ FWHM (Fig. 1e) while the FWHM is twice larger (Fig. 1c,g) without plasmonic effect. The proposed plasmonic structure definitely exposes an enhanced self-focusing nonlinearity. The strong light confinement is due to the presence of the plasmon-soliton wave that propagates in the studied structures only for the TM polarization. Different configurations have been characterized and modeled as a function of beam input intensity that confirm the formation of these plasmon-soliton waves [6].

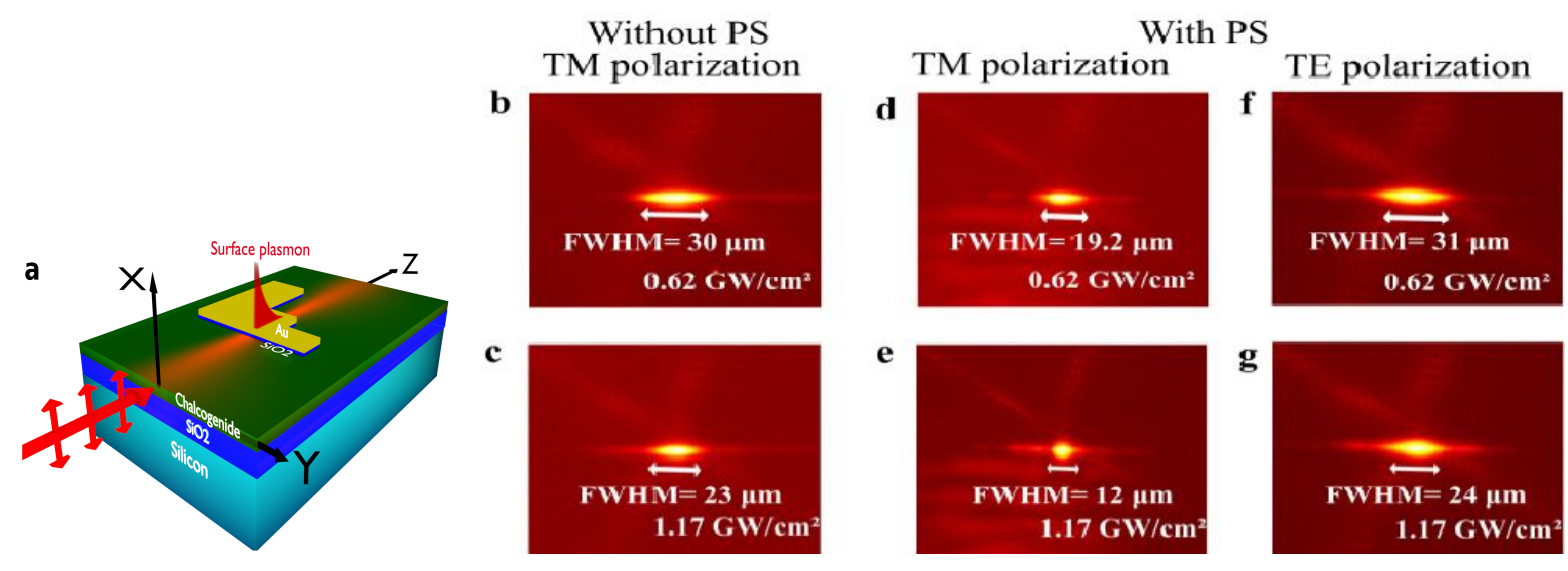

Figure 1: Plasmon-soliton wave experiment. a: Scheme of the structure. b,c,d,e,f, and g: Observed self-focusing effect for three configurations: without plasmonic structure (PS) for TM mode (b and c), with PS for TM mode (d and e) and for TE mode (f and g) at an input intensity of $0.62 \mathrm{GW} / \mathrm{cm} 2$ (first row) and $1.17 \mathrm{GW} / \mathrm{cm} 2$ (second row).

\section{References}

[1] V. M. Agranovich, V. Babichenko, and V. Y. Chernyak, "Nonlinear surface polaritons," JETP Lett 32, 512515 (1980).

[2] J. Ariyasu, C. T. Seaton, G. I. Stegeman, A. A. Maradudin, and R. F. Wallis, "Nonlinear surface polaritons guided by metal films," J. Appl. Phys. 58, p. 2460 (1985).

[3] E. Feigenbaum and M. Orenstein, "Plasmon soliton,” Opt. Lett. 32(6), p. 674 (2007)

[4] W. Walasik, V. Nazabal, M. Chauvet, Y. Kartashov, and G. Renversez, "Low-power plasmon-soliton in realistic nonlinear planar structures," Opt. Lett. 37(22), p. 4579 (2012).

[5] M. M. R. Elsawy and G. Renversez, "Exact calculation of the nonlinear characteristics of 2D isotropic and anisotropic waveguides", Opt. Lett., vol. 43(11), p. 2446, (2018).

[6] T. Kuriakose, G. Renversez, V. Nazabal, M. Elsawy, T. Halenkovic, P. Nĕmec, and M. Chauvet, "Experimental demonstration of plasmon-soliton coupling”, arXiv:1811.06525 [physics.optics], (2018). 